\title{
Digital Transformation for Efficient Communication in the Workplace: Analyzing the Flow Coworking Tool
}

\author{
Hyun Young Cho' ${ }^{1}$, Hak-June Lee ${ }^{2}$ \\ ${ }^{1}$ Dongduk Women's University, Seoul, Korea \\ ${ }^{2}$ Madras Check, Seoul, Korea
}

Objectives: The coronavirus disease (COVID-19) pandemic has necessitated new ways of conducting business, including hybrid work, which requires the active digital transformation of workplaces. This study examined cases in which the coworking tool Flow was adopted and explained Flow's characteristics and user experiences.

Methods: Flow is a coworking tool developed by Madras Check to improve communication efficiency between workers. This case report was based on interviews and surveys of Flow users.

Results: Flow was designed to increase productivity related to tasks and efficient management. According to client interviews, surveys, and our case analysis, Flow appears to achieve its purpose. In a survey of 1,429 respondents, Flow was found to reduce the amount of time spent checking messages via email and messenger applications, and respondents felt that Flow was a user-friendly tool and were willing to recommend Flow to others.

Conclusions: The present case report shows how the Flow coworking tool contributed to fostering a digital work environment. Our analysis of the benefits and user experiences of Flow indicates that such coworking tools are necessary for digital transformation so that organizations can create a hybrid work environment, which is the primary work format following the COVID-19 pandemic.

Key Words: Flow, Coworking Tool, Digital Transformation, Hybrid Work, Virtual Office

\section{Introduction}

According to an analysis by Trend Korea 2022 (Kim et al., 2021), four distinctive social trends are expected to arise in 2022, and these trends are likely to be strengthened due to the coronavirus disease 2019 (COVID-19) pandemic. The trends are 1) nano society, 2) rustic lifestyles, 3) the extended presence

Received: Dec 2, 2021 Revised: Dec 13, 2021 Accepted: Dec 28, 2021 Corresponding author: Hyun Young Cho

Department of Business Administration, Dongduk Women's University, 60 Hwarang-ro 13-gil, Seongbuk-gu, Seoul 02748, Korea

Tel: +82-2-940-4423, E-mail: hycho12@dongduk.ac.kr

This is an Open Access article distributed under the terms of the Creative Commons Attribution Non-Commercial License (http://creativecommons.org/licenses/ by-nc/4.0/) which permits unrestricted non-commercial use, distribution, and reproduction in any medium, provided the original work is properly cited.

Copyright ( $\odot 2022$ Korean Association for Business Communication. of technology, and 4) the reemergence of Generation X. The nano society trend refers to the phenomenon of widespread individualism due to an increase in smartphone, social media, and algorithm usage; this trend is becoming further intensified by the COVID-19 pandemic. Second, the rustic lifestyle trend refers to individuals' ability to pursue a leisurely lifestyle as if they lived in a rural area even when residing in a city. Third, the extended presence of technology trend refers to the increased use of live commerce and metaverse technologies. Finally, Generation $\mathrm{X}$, the members of which experienced both the analog and digital era, has emerged as a core group with a high degree of purchasing power. Each of these four distinctive trends can inspire changes in the way business is conducted. Due to deepening individualism, employee evaluations will increasingly be based on individual achievement rather than cooperative ability. The increasing preference for a rustic lifestyle will lead to a 
wider variety of workplaces in addition to a typical office setting and flexible working time. In addition, the extended presence of technology enables new types of workplaces through the use of metaverse-based coworking tools and virtual offices. Finally, members of Generation $\mathrm{X}$ are now old enough to be middle managers, connecting Millennials and members of Generation $\mathrm{Z}$ to older generations in organizations and enhancing communication between them.

In response to such changes in the business environment, many firms are trying to change the work environment. For example, global companies such as Google and Apple have extended telecommuting or work-from-home policies in response to the COVID-19 pandemic, and Korean firms such as Naver Line and Hyundai have begun to implement hybrid work models. In an analysis of 61 major listed corporations in the US, MarketWatch reported that $69 \%$ of them planned to adopt a hybrid work model. The proportion of companies in South Korea that used a hybrid work model increased from $4 \%$ in 2015 to $15 \%$ in 2020 (Wiltermuth, 2021).

Along with these shifts in work trends in response to the COVID-19 pandemic, companies now understand the need to reestablish their overall business strategies beyond temporary measures in response to the pandemic, as long-term changes are taking place in the business environment. Hybrid work is one of the work trends in 2022 that is guiding new business strategies (Offin, 2022). Hybrid work combines traditional office-based working with remote work that has been widely used after COVID-19, and $83 \%$ of people wanted to work in hybrid format after pandemic according to one study in May 2021 (Christian, 2022). Hybrid work enables the flexible use of digital technology and company resources and allows employees to experience work from any place at any time. Hybrid work enables an increase in the flexibility of the work environment as well as employee autonomy concerning decision-making. Hybrid work also allows companies to retain teams and human resources for productive cooperation, enabling new opportunities for growth.

As a result, hybrid work has been suggested as a new form of work. For example, Gartner Top Strategic Technology Trends for 2022 predicted that $75 \%$ of the companies that implement hybrid work will have a $25 \%$ faster growth rate than their competitors by 2023. In addition, a predicted 9 out of 10 companies will combine remote work and fieldwork in the future (Lund, Madgavkar, Manvika, \& Smit, 2021), and Global Workplace Analytics (2022) predicted that $70 \%$ of all human resource workers will work from home by 2025.

As such, the COVID-19 pandemic has increased the need for new ways of conducting business, such as hybrid work. Hybrid work calls for active digital transformation in the workplace.
There are several ways to increase productivity under hybrid work environment, and digital transformation is one of them (Pinola, 2021). Specifically, it was suggested that workers should go paperless as much as possible, without getting around the hassle of hauling of paper files, create schedule managers for efficient time management, and stay connected with co-workers for efficient hybrid work (Pinola, 2021). These suggestions are closely related to digital transformation in the organization, which can be enabled by coworking tools where workers can simultaneously communicate on the given project without constrained by time and place. Thus, it appears that COVID-19 pandemic facilitates the need for coworking tools. In fact, it has been reported that coworking tool market is expected to grow from $\$ 47.2$ billion in 2021 to $\$ 85.8$ billion in 2026, recording a compound annual growth rate of $12.7 \%$ (Kim, 2021).

The present case report analyzes the coworking tool Flow, developed by Madras Check, to help prepare companies for digital transformation. In the next section, we describe the background concerning companies' shift to digital transformation in greater detail and introduce the key features and benefits of Flow. Next, we describe the experiences of companies that implemented Flow and discuss the implications of digital transformation for business strategies.

\section{Case Description}

\section{Coworking Solutions Proposed by Flow}

Flow is a coworking tool developed by Madras Check that helps companies digitally transform the work environment. Flow refers to the experience of immersion in a task, enabling the user to proceed toward a goal. Madras Check created Flow to help workers concentrate on tasks without being overwhelmed by so many communication routes. Approximately 3,000 companies, ranging from large to small in size, have adopted Flow, and approximately 200 companies newly adopt Flow each month. As such, Flow ranks first among similar coworking tools, and the demand for Flow has increased by three times since the beginning of the COVID-19 pandemic in January 2020.

Flow has several distinctive features that differentiate it from its competitors. First, employees can create project rooms based on individual project topics and invite related personnel for timeline-based collaboration like Facebook. Flow provides not only messenger services but also posting-based cooperation tools. Though communication through messenger is convenient, it can be hard to continuously keep track of messages. In contrast, posting-based communication makes it easier to keep track of messages and feedback by enabling comments under posts that are relevant to ongoing projects. 
Since Flow is available on both PC and mobile, files can be opened using a mobile application, and large files can be shared without problems. In addition, Flow enables jobs to be tracked across five stages: "request," "in-process," "feedback," "completion," and "on hold." A powerful filtering function allows important tasks to be handled without error. Finally, Flow includes useful supplementary functions such as a smart calendar that workers can use to manage their schedules and the schedules of others, to-do lists, and strong security functions. Flow is an all-in-one coworking tool that can be operated intuitively in a manner that is familiar to Koreans; thus, anyone can easily use it without a long tutorial. Figure 1 and Figure 2 summarize Flow's distinctive features compared to other coworking tools and examples of how to use Flow for each job, respectively.

\section{Rules of Successful Coworking Fulfilled by Flow}

The use of Flow in a workplace can help fulfill the seven principles of successful coworking, which include place, connection, real-time tracking, objectives, security, partnership, and growth (Flow, 2021b).

\section{Place}

Flow can turn any location into a workplace in seconds . For successful collaboration to take place, there must be no restrictions on where an individual works. Flow provides a flexible workplace. LIG Nex1 was the first aerospace manufacturer and defense industry company to implement a work-fromhome policy, and flexible non-face-to-face work has progressed smoothly since the implementation of Flow.

\section{Connection}

It is crucial in a collaboration that all employees can communicate smoothly. Flow allows all employees to connect with each other while working in different locations and at different times. Flow unifies scattered communication channels via systematic linkage with existing in-house systems such as groupware, enterprise resource planning, mail, organization charts, and chatbots. In doing this, Flow strengthens communication by integrating data services provided by different and dispersed platforms into one platform (chatbot). The CEO of Busan-based GENTZ Bakery has stated that the use of Flow allowed GENTZ Bakery to enter Market Kurly, one of the largest online grocery services, stating that, while many tasks must be performed si-

\begin{tabular}{|c|c|c|c|c|c|}
\hline & \& flow & [] JANDI & NAVER WORKS & Thi Microsoft Teams & kakaowork \\
\hline Main Structure & Facebook timeline form & Group messenger & Messenger, Mail, \& Drive & Group messenger & Work Messenger \\
\hline Messenger & $\mathrm{O}$ & $\mathrm{O}$ & $\mathrm{O}$ & $\mathrm{O}$ & $\mathrm{O}$ \\
\hline Coworking Unit & Project & Channel & 0 & - & Group, Team \\
\hline To do List & $\begin{array}{l}\text { Strong to-do-list function } \\
\text { Designate the manager, and deadline }\end{array}$ & 0 & $x$ & $\begin{array}{c}\mathrm{X} \\
\text { (Need to use separate } \\
\text { service) }\end{array}$ & 0 \\
\hline Work Process & $\begin{array}{l}\text { 5-step real-time work process } \\
\text { Strong report function } \\
\text { Check the all employee's work process }\end{array}$ & $x$ & $x$ & $x$ & $x$ \\
\hline Calendar sharing & $\begin{array}{l}\text { Work schedule calendar (monthly/weekly/ } \\
\text { daily view), Location-based scheduling } \\
\text { (meetings, working outside,business trip), } \\
\text { Full schedule by the work deadline }\end{array}$ & $x$ & 0 & $x$ & $x$ \\
\hline Notification options & $\begin{array}{l}\text { Various functions as in Naver Band,'Do n } \\
\text { ot disturb; mode, Notification function for } \\
\text { only comments related to me }\end{array}$ & $\begin{array}{l}\text { On/Off only } \\
\text { per room }\end{array}$ & $\begin{array}{l}\text { Various notification } \\
\text { options for each room } \\
\text { \& Set Do Not Disturb }\end{array}$ & $\begin{array}{l}\text { Various notification } \\
\text { options for each room } \\
\& \text { Set Do Not Disturb }\end{array}$ & $\begin{array}{l}\text { Various notification } \\
\text { options for each room } \\
\& \text { Set Do Not Disturb }\end{array}$ \\
\hline $\begin{array}{l}\text { Inviting workers } \\
\text { outside the }\end{array}$ & $\begin{array}{l}\text { Collaboration with outsiders for ea } \\
\text { ch project on one screen }\end{array}$ & $\begin{array}{l}\text { limited available (must be } \\
\text { used as a team switch) }\end{array}$ & Limited available & Guest can be invited & Undefined \\
\hline Security Function & $\begin{array}{c}\text { Download/capture history can be checked/ } \\
\text { Strong security settings (Secondary } \\
\text { authentication, duplicate } \\
\text { login, capture/download prevention) }\end{array}$ & $\begin{array}{l}\text { Only download prevention } \\
\text { and download check } \\
\text { history prevention }\end{array}$ & $\begin{array}{l}\text { Data encryption/check } \\
\text { download history (download } \\
\text { limit/capture prevention } \\
\text { impossible) }\end{array}$ & account security & $\begin{array}{l}\text { Security features in } \\
\text { preparation }\end{array}$ \\
\hline Service format & $\begin{array}{l}\text { Rental method for small and medium-sized } \\
\text { enterprises (Cloud, SaaS) In-house method } \\
\text { for medium/large enterprises (On-Premise) }\end{array}$ & $\begin{array}{l}\text { Rental method for small } \\
\text { and medium-sized enter } \\
\text { prises (Cloud, SaaS) }\end{array}$ & Cloud type only & Cloud type only & $\begin{array}{l}\text { Rental method for small } \\
\text { and medium-sized } \\
\text { enterprises (SaaS) }\end{array}$ \\
\hline Characteristics & $\begin{array}{l}\text { Powerful workflow capabilities } \\
\text { Strong Functionality and Security } \\
\text { Project-driven social collaboration }\end{array}$ & Webhook function & $\begin{array}{l}\text { Large }(2 \mathrm{~GB}) \text { files } \\
\text { can be uploaded }\end{array}$ & Sharepoint connection & $\begin{array}{l}\text { Supports some functions } \\
\text { of groupware (attendance } \\
\text { management, electronic } \\
\text { approval) }\end{array}$ \\
\hline
\end{tabular}

Figure 1. Features of Flow compared to other coworking tools. 


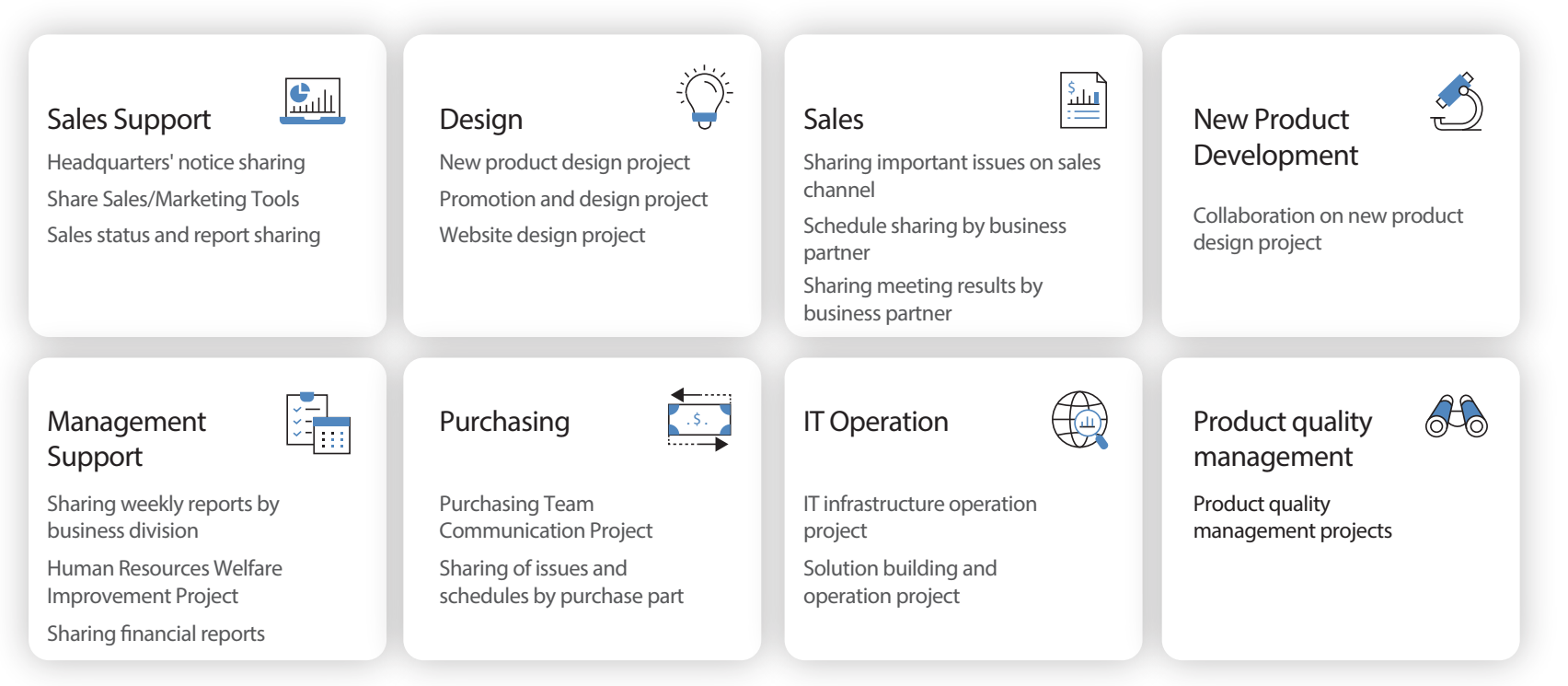

Figure 2. Application of Flow across various departments.

multaneously and promptly to run a bakery, the company was able to manage various projects systematically because of Flow.

\section{Real-Time Tracking}

Prompt decision-making is required in collaborative work. Flow provides workers with real-time tracking of the process of each task. Specifically, Flow has a function to assign the priority level of tasks, the person in charge, and the deadline for each task. This allows for rapid decision-making and business processing.

\section{Objectives}

Flow allows each employee to determine the overall objective of the organization, which in turn enables them to create detailed task goals based on the overall objective.

\section{Security}

Due to the development of information technology, the speed and convenience of conducting business have improved; however, document sharing and data leakage have become easier, and even a single USB can cause great damage. Flow has a strong security system with various functions including a screen capture prevention setting, 2-factor authentication, a duplicate login limit, and data encryption that minimize the possibility of such issues. Hyundai Mobis, one of Flow's clients, secures information by requiring employees to agree to an information protection pledge when creating a new project and penalizes those who violate this pledge. In addition, Flow's text security authentication procedure severely restricts login by non-members.

\section{Partnership}

With Flow, organizations can invite an unlimited number of partners for free, allowing for close and systematic collaboration. For example, POSCO and E-LAND invited marketing agencies using Flow to systematically collaborate on marketing/ advertisement-related projects.

\section{Growth}

Flow can store all project-related data indefinitely, so workers can easily find the data they want at any time. Thus, even members who join a project late can refer to the project history. In addition, data from past projects can help users determine what worked and what did not, providing the opportunity to grow. COOCON, a company that provides a fintech API platform and business information, strongly recommended Flow based on its experience of growth after COOCON was able to enhance the quality of its core customer-oriented products using the work history stored by Flow.

\section{Benefits of Flow Based on a Survey}

In this section, we examine the results of a survey conducted by Flow of 1,429 workers (Flow, 2021d) and discuss the benefits of Flow. First, after using Flow, the average scores for the quality of collaborative tasks and the management of each worker's performance increased from 58 to 80 and 58 to 89 , respectively (Figure 3). Thus, Flow enhanced two of the basic principles of coworking-productivity and management efficiency-by enabling business to be conducted in innovative ways. Second, 
before after

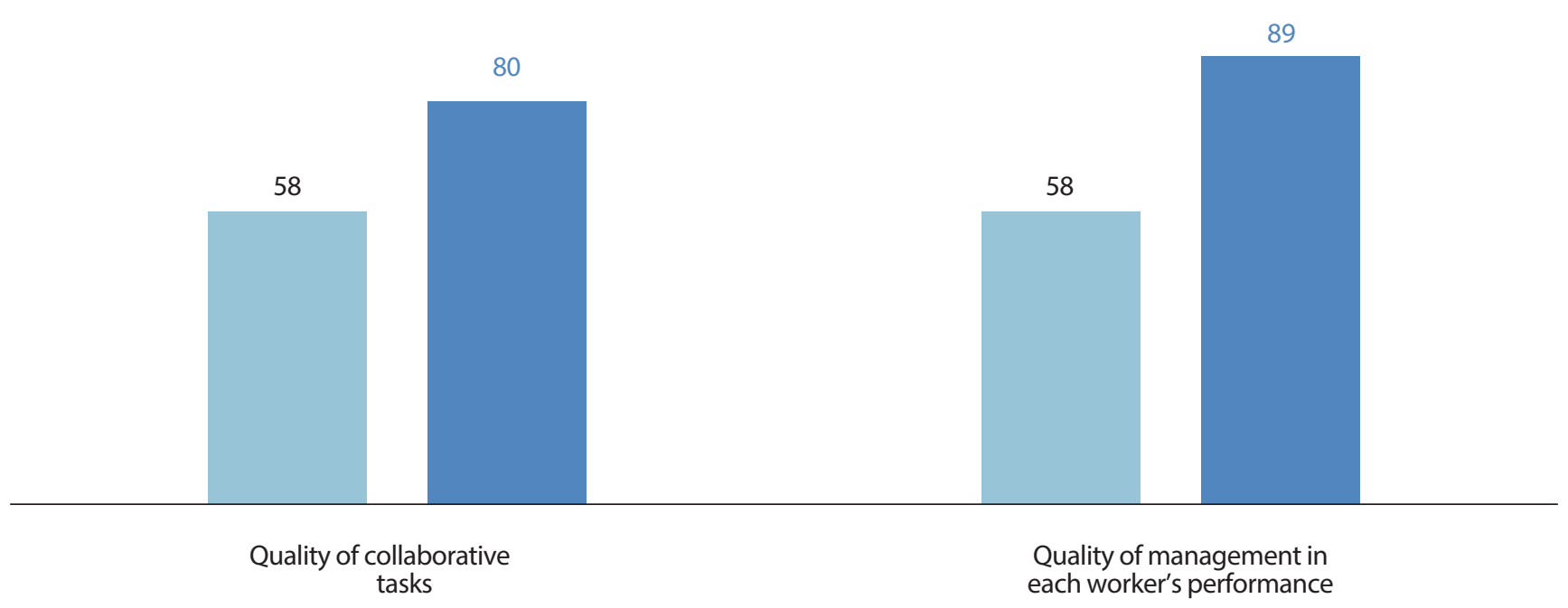

Figure 3. Improvement of quality in collarative tasks and performance management.

survey respondents found that Flow helped reduce wasted time spent on tasks that were unrelated to workers' main tasks.

Specifically, before using Flow, $40.6 \%$ of the respondents felt that about 30 minutes to 1 hour per day was wasted due to inefficient collaboration methods used before the implementation of Flow. In particular, the respondents said that they wasted a lot of time replying to messages over messenger applications and emails. After the implementation of Flow, 38.2\% of the respondents said that approximately 30 minutes to 1 hour per day was saved (Figure 4). Finally, the average overall satisfaction score exceeded 4 out of 5 points. Specifically, the average ratings for "ease of operation," "operation speed," "service function," and "user-friendliness" were 4 points, 4 points, 4.1 points, and 4.1 points, respectively, and $83 \%$ of the respondents said that they were willing to recommend Flow to others (Figure 5). Overall, wasted time was greatly reduced through the use of Flow, and the efficiency of collaboration increased.

\section{Instances of Digital Transformation after the Adoption of Flow}

Case A

Company A adopted Flow after searching for a coworking tool that could be connected to both PC and mobile devices. Initially this company has used Jira and Conflience but they are suitable only for those who work in R \& D department. What Company A has looked for is the toolthat workers across the whole organization could use regardless of whether they worked in the R\&D department. Since personnel information is automatically connected to Flow, the handling of employees' on- and off-boarding information was automated. In addition, the company's performance appraisal system connected to Flow so that workers could register their work output and track their work history. Thus, workers could seamlessly communicate with their colleagues using Flow and simultaneously post their work outputs to receive and share feedback.

\section{Case B}

Comapny B adopted Flow for two reasons. First, the company found communication through messenger and email to be inefficient, which led them to adopt an Objectives and Key Results (OKR) system. The company then searched for a coworking tool that could be connected to the OKR system and subsequently adopted Flow. Second, as the format of work shifted toward a hybrid work model, the company realized that performance appraisals, as well as communication channels, should be revised in an innovative way. Workers now communicate through Flow, and their work outputs are reported using the OKR system. Flow was also connected to Company B's video conference system, Nos.

\section{Case C}

Company $\mathrm{C}$ adopted Flow to enhance work efficiency and transparently evaluate employees' work performance. In a faceto-face work model, it was easy to evaluate individual performance, but it became difficult to evaluate an individual's work performance in a non-face-to-face work setting. Therefore, this company chose Flow as a coworking tool to help track workers' performance in a non-face-to-face context. The telecommuting daily work record system used by the company was connected with Flow so that each individual's work history was automat- 
How much time per day do you think is wasted in inefficient collaboration?

More than 2 hrs. (4.9\%)

$1.5 \sim 2$ hrs. (7.6\%)

1 hr. 1.5 hrs. (23.5\%)

30mins 1hr. (40.6\%)

Less than 30 mins

(23.4\%)

\section{0 mins. 1 hr. $40.6 \%$}

$23.4 \%$

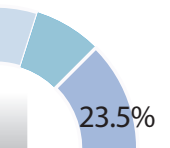

$23.5 \%$

Figure 4. Perceived efficiency with Flow tool.

Satisfaction with Flow (out of 5)

Overall average of service satisfaction: " 4 or higher"

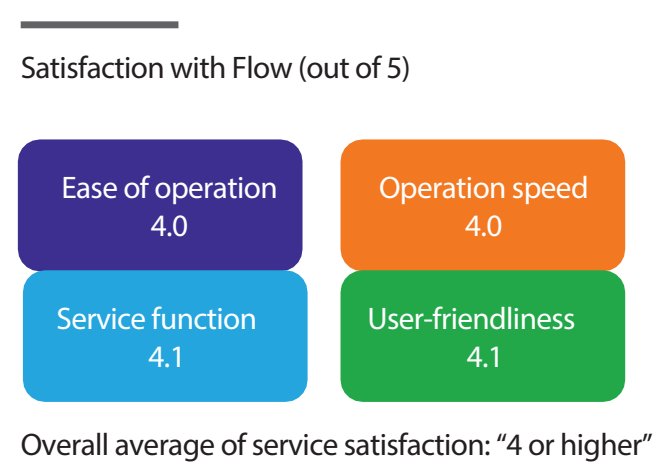

Figure 5. Overall satisfaction with Flow.

ically recorded and synced to Flow, enabling each employee to easily share what they do with other employees. In addition, employees could open small rooms in Flow to communicate with each other and form groups to interact with Flow as a small community.

\section{Case D}

Company D adopted Flow to efficiently develop and operate information technology projects with its R\&D department and partners. In addition to collaboration, Flow was used to enhance security related to mobile device management. In particular, company's own Mobile Device Management (MDM) solution is connected to Flow so that the internal security system is advanced. Also, this comapny customized the security system in Flow so that a warning notification appeared when sensitive information such as an individual's social security number or account number was entered while workers chatted or shared files.
How much time do you feel you have sav ed per day through Flow?
Willingness to recommend to others

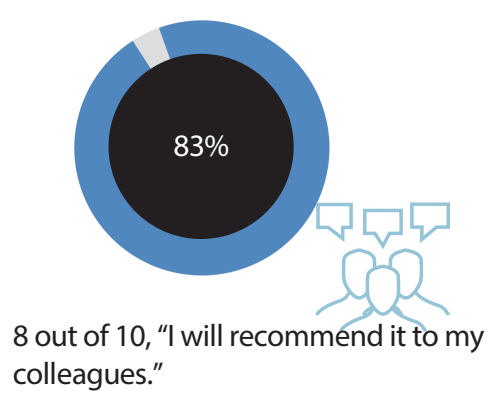

More than 2 hrs. (3.6\%)

$1.5 \sim 2$ hrs.(5.3\%)

1hr. 1.5 hrs.(15.9\%)

30mins. 1hr.(38.2\%)

Less than 30 mins (37\%)

30 mins 1hr. $38.2 \%$

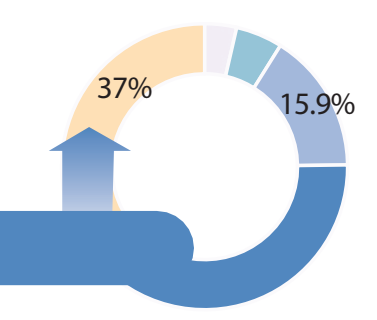

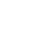


it is used not only for all employees in the head office, but also for collaboration with partners. Flow was connected with the groupware, schedule management, and email applications used by this company.

\section{Case G}

Company $\mathrm{G}$ introduced Flow to reorganize a smart communication platform that can be used with the next-generation version of ERP. The second reason for introducing Flow is to efficiently communicate with many stores branches across the country and to manage the performance of those branches. This company linked the chat function of Flow with SAP ERP, which was used previously, to enable inventory inquiry and sales inquiry through Flow's chatbot.

In addition, Flow is installed and operated in AWS private cloud.

\section{Discussion}

This case report showed how the Flow coworking tool contributed to companies' attempts to establish digital work environments by describing the characteristics of Flow and companies' experiences using Flow. The need for coworking tools has increased due to growing uncertainty about how to best conduct business in the modern era and was accelerated as a result of the COVID-19 pandemic (Schubert \& Williams, 2022). Approximately $87 \%$ of companies have not determined a business plan for the next year due to business uncertainty (KDI Economic Policy Uncertainty, 2020), and 56.2\% of 80 major companies and 236 small and medium-sized companies have not yet established investment plans (Korea Chamber of Commerce and Industry, 2021).

As uncertainty in business environments grows, businesses must stick to the basics-productivity and management efficiency. In addition, innovation is required to promptly respond to uncertainties in the business environment. Introducing a coworking tool is a way to prioritize these basic rules in innovative ways. Hybrid work is an example of an innovation related to how work is conducted. In the COVID-19 era, during which non-face-to-face work environments are dominant (de Lucas Ancillo, del Val Núñez, \& Gavrila, 2021; Newman \& Ford, 2021), hybrid work presents a suitable work format for this context (Yang, Kim, \& Hong, 2021). Implementing a hybrid work model requires a digital transformation in organizations (Gabryelczyk, 2020; Soto-Acosta, 2020), and the need for digital transformation often facilitates the adoption of coworking tools.

An increase in demand for coworking tools is inevitable since communication tools have become more varied since the late 2010s. From the 1980s to early 2010s, the major communication tools used in workplaces were emails and PC messenger applications, while groupware, video (Zoom conferencing and remote meetings), and metaverse applications have become more prevalent beginning in the late 2010s. As a result, employees often answer phone calls while replying to messages and emails, which increases the complexity of their work and reduces work efficiency (Flow, 2021a). In order to enhance work efficiency, coworking tools have been developed. The usefulness of coworking tools for enhancing smooth communication and work efficiency is supported by the existing literature.

Since the COVID-19 pandemic, more people have begun to work from home, reducing employee engagement, which is one of the most prominent issues for human resource managers in the COVID-19 era (Chanana \& Sangeeta, 2020). Specifically, working from home makes it difficult to focus compared to working in an office setting. In addition to interruptions by family members and anxiety about the inability to separate work from life, some employees do not have access to the equipment needed to complete their work at home. Because of these factors, telecommuting can reduce employee engagement (Chanana \& Sangeeta, 2020).

In response to this issue, various engagement practices have been introduced in organizations such as virtual learning and development, online team-building activities, webinars by industry experts, online weekly alignment sessions, and team meet-ups over video conferences during lunch. Many of these activities have been successful, helping employees learn new skills and grow and increasing employees' work productivity and sense of commitment (Chanana \& Sangeeta, 2020). Coworking tools can also be understood as tools for engagement since they increase users' motivation to collaborate and increase work efficiency.

In addition, in response to the acceleration of digital transformation caused by the COVID-19 pandemic, many believe that working digitally will be the dominant work format in the future (Nagel, 2020). Our case analysis of companies across various industries revealed that working in a virtual office setting using Flow helped improve business efficiency, supporting Nagel's (2020) prediction. Finally, the notion of the workplace has changed from a building or place where people perform their job to a wider concept encompassing more dimensions such as social relationships, gathering, training, motivation, and engagement (Fisher, 2010; Gill, 2019). Therefore, a virtual office setting that addresses these various dimensions is important. Coworking tools that enable work in a virtual office setting will increase in relevance in the coming years.

Coworking tools like Flow enable companies to fulfill the 
three requirements for successful implementation of a hybrid work model: making digital tools available to foster a flexible work environment, improving the efficiency of work processes, and cultivating an innovative organizational culture (Flow, 2021c). Based on the survey and case analysis, Flow helped to change the overall work environments of organizations in an innovative way. Specifically, Flow enabled seamless communication between employees regardless of location. Instead of going to an office to check and respond to emails, workers became capable of communicating using mobile devices and in real-time with Flow.

In addition, employees who worked on the same project could share their objectives and work in accordance with those objectives, and external partners could easily be invited to join the project. Flow's strong security functions enabled workers to keep important information safe, and by tracking past work history, workers could learn from past successes and failures and use data on Flow as a tool for growth. Therefore, Flow contributes to innovation in workplaces undergoing a digital transformation. Based on this case analysis, companies across various industries can benefit from using Flow. By using Flow, the companies in the present study agreed that communication between colleagues was smoother, work efficiency increased, and transparency with regard to employee evaluations improved since one's work could be monitored in real-time. In the future case report, it will be interesting to examine in detail how Flow can be differentiated when compared with competitor's coworking tools such as Notion, Base Camp, and Jira.

\section{Conclusion}

Coworking tools are expected to lead to changes in work processes that are markedly different from previous ones. Specifically, in the past, one-way communication via phone or email in physical spaces was the dominant work format, and collaboration was conducted as teams. Using coworking tools, however, anyone can freely communicate in both directions in an online virtual space, and organizations can conduct business as project units rather than team units. In order to build such work processes, both employees and managers need to cultivate an organizational culture with a digital mindset. Based on this case analysis of Flow, it can be concluded that coworking tools are necessary for the digital transformation of organizations. Companies should actively adopt coworking tools in response to the current need to cultivate a hybrid work environment. Such digital transformation efforts in which people can perform their jobs without time or place constraints will ultimately increase the autonomy and productivity of workers.

\section{References}

Chanana, N., \& Sangeeta (2020). Employee engagement practices during COVID-19 lockdown. Journal of Public Affairs, 21(4), e2508.

Christian, A. (2022, January 21). A part-remote, part-office schedule has been hailed as the future of work. Yet in this hybrid set-up, some employees have never been so tired. Retrieved from https:// www.bbc.com/worklife/article/20220120-why-hybrid-work-isemotionally-exhausting\#: :text=In\%20theory\%2C\%20hybrid\%20 offers $\% 20$ the,for\%20focused $\% 20$ work\%20at\%20home

de Lucas Ancillo, A., del Val Núñez, M. T., \& Gavrila, S. G. (2021). Workplace change within the COVID-19 context: A grounded theory approach. Economic Research-Ekonomska Istraživanja, 34(1), 2297-2316.

Fisher, C. D. (2010). Happiness at work. International Journal of Management Reviews, 12(4), 384-412.

Flow (2021a, May 20). Coworking tools, essential conditions for companies to achieve their goals. Retrieved from https://post. flow.team/insight/4505

Flow (2021b, June 7). 10 Construction strategies for 2021 digital work trends. Retrieved from https://post.flow.team/insight/5005

Flow (2021c, June 21). Defining coworking tool and strategy for constructing 3 components of digital transformation. Retrieved from https://post.flow.team/insight/5262

Flow (2021d, August 26). 2021 Flow user report. Retrieved from https://post.flow.team/tip/6392

Gabryelczyk, R. (2020). Has COVID-19 accelerated digital transformation? Initial lessons learned for public administrations. Information Systems Management, 37(4), 303-309.

Gartner (2022). Gartner top strategic technology trends for 2022. Retrieved from https://www.gartner.com/en/information-technology/insights/top-technology-trends

Gill, M. J. (2019). The significance of suffering in organizations: Understanding variation in workers' responses to multiple modes of control. Academy of Management Review, 44(2), 377-404.

Global Workplace Analytics. (2022). Key telecommuting data and trends. Retrieved from https://globalworkplaceanalytics.com/ telecommuting-statistics

KDI Economic Policy Uncertainty (2020). EPU index. Retrieved from https://eiec.kdi.re.kr/issue/epuList.do

Kim, D. H. (2021, July 15). Corporate collaboration market to grow at a CAGR of $12.7 \%$ by 2026 . Retrieved from https://www.ciokorea.com/news/201129

Kim, N. D., Chun, M. Y., Choi, J. H., Lee, H. E., Lee, J. Y., Lee, S. J., ... Lee, H. W. (2021). Trend Kore 2022. Seoul, Korea: Window of Future.

Korea Chamber of Commerce and Industry [KCCI] (2021, No- 
vember 8). With COVID-19 era business environment prospects and response tasks investigation. Retrieved from http://www. korcham.net/nCham/Service/Economy/appl/KcciReportDetail. asp?SEQ_NO_C010=20120934352\&CHAM_CD=B001

Lund, S., Madgavkar, A., Manyika, J., \& Smit, S. (2020, November 23). What's next for remote work: An analysis of 2000 tasks, 800 jobs, and nine countries. McKinsey Global Institute. Retrieved from https://www.mckinsey.com/featured-insights/future-ofwork/whats-next-for-remote-work-an-analysis-of-2000-tasks800 -jobs-and-nine-countries

Nagel, L. (2020). The influence of the COVID-19 pandemic on the digital transformation of work. International Journal of Sociology and Social Policy, 40(9/10), 861-875.

Newman, S. A., \& Ford, R. C. (2021). Five steps to leading your team in the virtual COVID-19 workplace. Organizational Dynamics, 50(1), 100802.

Offin, N. (2022, January 5). Hybrid working: What to expect in 2022. IDG Connect. Retrieved from https://www.idgconnect.com/article/3641337/hybrid-working-what-to-expect-in-2022.html
Pinola, M. (2021, July 26). 6 productivity tips for your new hybrid work life. Retrieved from https://www.nytimes.com/wirecutter/ blog/productivity-tips-for-hybrid-work/

Schubert, P., \& Williams, S. P. (2022). Enterprise collaboration platforms: An empirical study of technology support for collaborative work. Procedia Computer Science, 196, 305-313.

Soto-Acosta, P. (2020). COVID-19 pandemic: Shifting digital transformation to a high-speed gear. Information Systems Management, 37(4), 260-266.

Wiltermuth, J. (2021, October 9). Nearly 70\% of big U.S. companies are adopting a flexible work model, says Morgan Stanley. Market Watch. Retrieved from https://www.marketwatch.com/story/ nearly-70-of-big-u-s-companies-are-adopting-a-flexible-workmodel-says-morgan-stanley-11633467831

Yang, E., Kim, Y., \& Hong, S. (2021). Does working from home work? Experience of working from home and the value of hybrid workplace post-COVID-19. Journal of Corporate Real Estate. doi: 10.1108/JCRE-04-2021-0015. 